\title{
Recent Results on Charmonium Transitions studied with BESIII
}

\author{
Olga Bondarenko* $\dagger$ \\ KVI, University of Groningen \\ E-mail: o.bondarenko@rug.nl
}

Charmonium spectroscopy is an ideal tool to provide insight into the non-perturbative dynamics of the strong force. The charmonium system has been systematically studied with the BESIII spectrometer operated at the BEPCII electron-positron collider at IHEP Beijing, China. We present BESIII results on radiative and hadronic transitions between charmonium states below the opencharm threshold. These transitions have been exploited to study charmonium resonances, such as the $\eta_{c}$ and the $\eta_{c}^{\prime}$. In addition, we report on improved measurements of branching fractions for isospin-violating decays, which will be essential to determine the light-quark mass ratio.

Sixth International Conference on Quarks and Nuclear Physics April 16-20, 2012

Ecole Polytechnique, Palaiseau, Paris

\footnotetext{
* Speaker.

${ }^{\dagger}$ on behalf of the BESIII Collaboration.
} 


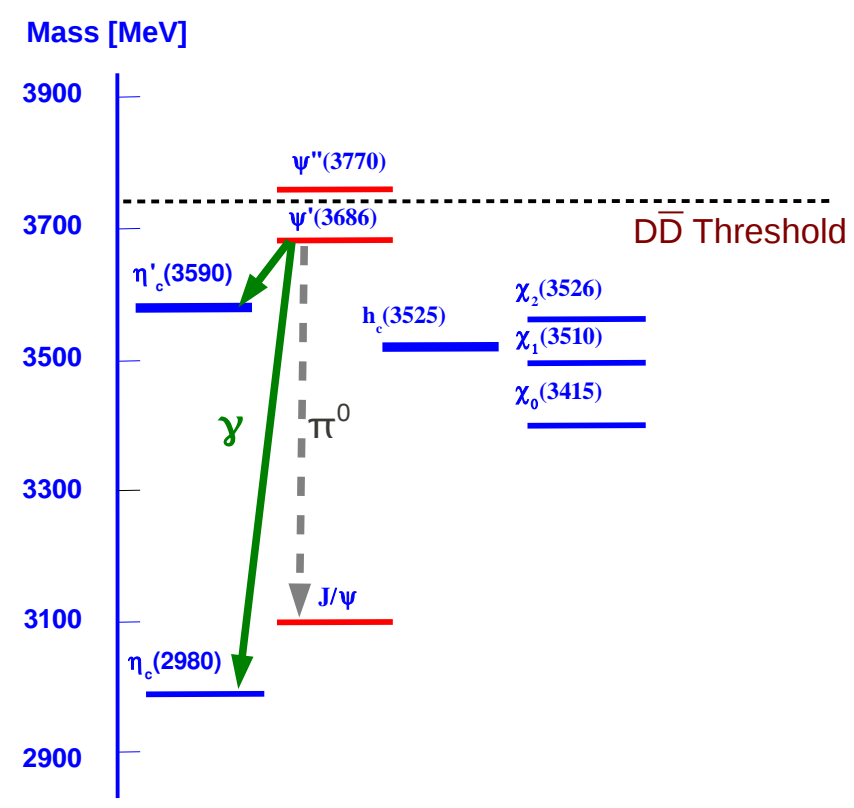

Figure 1: The spectrum of the low-lying charmonium mesons. The black dashed line indicates the opencharm threshold. The green arrows indicate transitions used for the $\eta_{c}$ and $\eta_{c}^{\prime}$ mass and width measurements. The gray dashed line indicates the isospin-violating transition $\psi^{\prime} \rightarrow \pi^{\circ} J / \psi$.

\section{Introduction}

Despite the successes of the Standard Model, the QCD-based nature of the strong interaction is insufficiently understood. Charmonium spectroscopy is an ideal tool to rigorously study the structure of the strong force. In particular, a precise determination of the properties of charmonium states and their transitions provides strong guidelines to various theoretical approaches, such as lattice calculations and effective field theories, thereby, gaining insight into the quark confinement and the generation of hadron masses.

The BESIII experiment [1] at the BEPCII $e^{+} e^{-}$collider in Beijing obtained the world's largest data sets of charmonium states at center-of-mass energies corresponding to the $J / \psi, \psi^{\prime}$ and $\psi^{\prime \prime}$ vector-meson masses. The precision of various resonance parameters and decay properties of charmonium states has been improved significantly by BESIII with respect to previously published data. Furthermore, rare charmonium transitions have been successfully observed for the first time by the BESIII collaboration [2] [3].

In this paper, we present a fraction of the recently obtained results with BESIII. This includes the observation of the magnetic-dipole transition (M1) from the $\psi^{\prime}$ to the charmonium ground-state $\eta_{c}$ and to its first radial excitation the $\eta_{c}^{\prime}$. In addition, we report on an improved measurement of the relative branching fraction between the isospin-forbidden decay $\psi^{\prime} \rightarrow J / \psi \pi^{\circ}$ and the $\psi^{\prime} \rightarrow J / \psi \eta$ reaction. The corresponding transitions are depicted in Fig. 1. 


\section{Measurement of the mass and width of the $\eta_{c}$}

Surprisingly, the properties such as the mass and width of the ground state of charmonium, the $\eta_{c}$, are still poorly understood both experimentally and theoretically. The particle data group (PDG) [4] has listed more than 20 measurements of the $\eta_{c}$ mass with a world average value of $2980.3 \pm 1.2 \mathrm{MeV}$. The precision is significantly worse than that for the $\psi^{\prime}$ or $J / \psi$ mesons. Moreover, significant disagreements among the measurements are observed. The experimental situation on the total width of the $\eta_{c}$ is even worse. The PDG reported a world-average value of $\Gamma=28.6 \pm 2.2 \mathrm{MeV}$ with large discrepancies between the results of various types of measurements, thereby, strongly depending on the production process of the $\eta_{c}$.

The BESIII collaboration recently published a precise measurement of the mass and width of the $\eta_{c}$ [5]. The $\eta_{c}$ mesons were produced via the M1 radiative transition $\psi^{\prime} \rightarrow \gamma \eta_{c}$ with an exclusive measurement of the decay of the $\eta_{c}$ into six final states, namely $\eta_{c} \rightarrow X_{i}$, where $X_{i}=$ $K_{s} K^{+} \pi^{-}, K^{+} K^{-} \pi^{\circ}, \eta \pi^{+} \pi^{-}, K_{s} K^{+} \pi^{+} \pi^{-} \pi^{-}, K^{+} K^{-} \pi^{+} \pi^{-} \pi^{\circ}$ and $3\left(\pi^{+} \pi^{-}\right)$, where $K_{s} \rightarrow \pi^{+} \pi^{-}$ and $\pi^{\circ}(\eta) \rightarrow \gamma \gamma$. Distinct $\eta_{c}$ signals are seen in each of the six channels. The mass spectrum for the $K_{S} K^{+} \pi^{-}$channel is shown in Fig. 2. For all the observed channels, the $\eta_{c}$ signal has a distinctively asymmetric shape with a long tail at low masses and a rapid drop on the high mass side. This observation suggests a possible interference with a coherent non-resonant background. A simultaneous fit for the $\eta_{c}$ mass and width was performed on all the mass spectra, where the interference between $\eta_{c}$ and non-resonant decays is considered and the quantum numbers of the non- $\eta_{c}$ components are assumed to be $0^{-+}$. Assuming a universal relative phase between the two amplitudes, an $\eta_{c}$ mass of $M=2984.3 \pm 0.6 \pm 0.6 \mathrm{MeV} / c^{2}$ and a width of $\Gamma=32.0 \pm 1.2 \pm 1.0 \mathrm{MeV}$ were obtained.

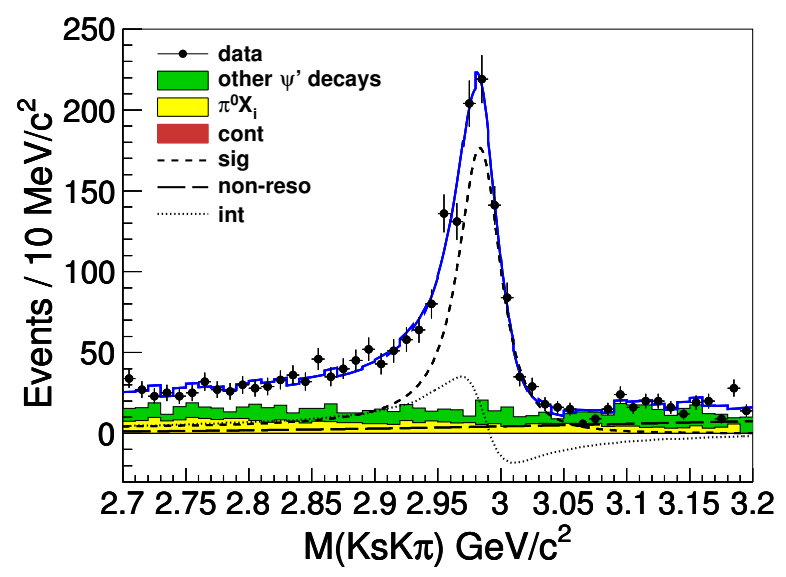

Figure 2: The mass spectra for decay mode $\eta_{c} \rightarrow K_{s} K^{+} \pi^{-}$with the fit results superimposed. Points are data and the curve is the total fit result. The $\eta_{c}$-signal yield is shown as short-dashed line; the non-resonant component as a long-dashed line; and the interference between them as a dotted line. Shaded histograms in red (not visible on this histogram), yellow (light gray), and green (dark-gray), correspond to background contributions from the continuum, the $\pi^{\circ} K_{s} K^{+} \pi^{-}$channel, and other $\psi^{\prime}$ decays, respectively. The continuum background for this decay mode is negligible. 


\section{Observation of the M1 transition $\psi^{\prime} \rightarrow \gamma \eta_{c}^{\prime}$}

Among the charmonium states below the open-charm threshold, the $\eta_{c}^{\prime}$ meson has the most poorly measured mass and width. The PDG reported an average for the mass of $3637 \pm 4 \mathrm{MeV}$. The average width, based on two measurements, is $14 \pm 7 \mathrm{MeV}$.

BESIII observed for the first time the M1 transition, $\psi^{\prime} \rightarrow \gamma \eta_{c}^{\prime}$ [3], via the decay modes of $\eta_{c}^{\prime} \rightarrow K_{s} K \pi$ and $\eta_{c}^{\prime} \rightarrow K^{+} K^{-} \pi^{\circ}$, where $K_{s} \rightarrow \pi \pi$. Figure 3 shows the invariant mass, $M_{K_{S}^{\circ} K \pi}$, distribution which was obtained using a three-constraint kinematic fit for which the energy of the photon was allowed to float. The data are used to obtain measurements of the $\eta_{c}^{\prime}$ mass $M=$ $3637.6 \pm 2.9 \pm 1.6 \mathrm{MeV} / \mathrm{c}^{2}$ and the width $\Gamma=16.9 \pm 6.4 \pm 4.8 \mathrm{MeV}$, which are in a good agreement with the PDG values. The combined statistical signficance for the signal in the two modes is more than $10 \sigma$ under a wide range of assumptions about the signal and background properties and a branching product $B R\left(\psi^{\prime} \rightarrow \gamma \eta_{c}^{\prime}\right) \times B R\left(\eta_{c}^{\prime} \rightarrow K \bar{K} \pi\right)$ of $(1.30 \pm 0.2 \pm 0.30) \cdot 10^{-5}$ has been measured.

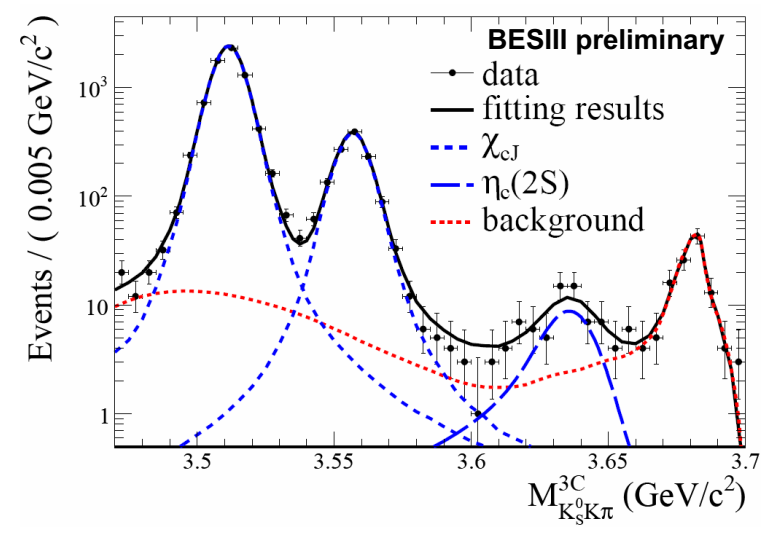

Figure 3: The $K_{s} K \pi$ invariant-mass spectrum for the radiative decay $\psi^{\prime} \rightarrow \gamma \eta_{c}^{\prime}$. Data are represented as filled circles together with the result of a likelihood fit (solid black line). The fit includes three resonances (blue dashed lines) and several other background sources (red dotted line).

\section{Measurements of branching fractions of isospin-violating transitions in charmonium}

The hadronic transitions $\psi^{\prime} \rightarrow \pi^{\circ}(\eta) J / \psi$ in the charmonium system were suggested to be a reliable source for the extraction of the ratio of up and down quark masses [6]. This was motivated by the observation that the contribution of the electromagnetic process was shown to be negligigle [7]. Based on a leading-order QCD multipole expansion, the relation between the ratio, $R_{\pi^{\circ} / \eta}$, of the branching fractions for the $\psi^{\prime} \rightarrow \pi^{\circ} J / \psi$ and $\psi^{\prime} \rightarrow \eta J / \psi$ transitions and the masses of the up and down quarks $\left(m_{u}, m_{d}\right)$ can be written as [8]:

$$
R_{\pi^{\circ} / \eta}=3\left(\frac{m_{d}-m_{u}}{m_{d}+m_{u}}\right)^{2} \frac{F_{\pi}^{2}}{F_{\eta}^{2}} \frac{M_{\pi}^{4}}{M_{\eta}^{4}}\left|\frac{\vec{q} \pi}{\vec{q} \eta}\right|^{3},
$$

where $F_{\pi(\eta)}, M_{\pi(\eta)}$ are the decay constants and masses of the $\pi / \eta$ mesons, respectively, and $\vec{q}_{\pi(\eta)}$ stands for the momenta of the corresponding particles produced in the transition $\psi^{\prime} \rightarrow \pi^{\circ}(\eta) J / \psi$ in the $\psi^{\prime}$ rest frame. Strikingly, the up-down quark-mass ratio obtained using Eq. (1) and data 
from the CLEO-c collaboration [9] is $m_{u} / m_{d}=0.40 \pm 0.01$, which is in discrepancy with other approaches, for example using the masses of the lightest scalar mesons [10]. Recently, it was suggested that the main source of this discrepancy may be due to the contribution of intermediate charmed-meson loops [11]. The light-quark mass ratio can only be extracted from these decays after establishing the effective field theory up to next-to-leading order. Experimental studies of all the isospin-violating transitions in the charmonium system will give an important input for the construction of the effective field theory.

Recently, the BESIII collaboration performed a new measurement of the ratio $R_{\pi^{\circ} / \eta}$, with $\pi^{\circ} / \eta \rightarrow \gamma \gamma$ and the leptonic decay mode of $J / \psi\left(J / \psi \rightarrow e^{+} e^{-} / \mu^{+} \mu^{-}\right)$. The reconstructed mass spectra of the $\pi^{\circ} / \eta$ together with background contributions are shown in Fig. 4 . The preliminary value of the ratio $R_{\pi^{\circ} / \eta}$ was found to be $(3.74 \pm 0.06 \pm 0.04) \%$, which is in a good agreement with the previously reported measurements [9] and its precision is significantly improved. The obtained result, together with other branching fractions of meson transitions in charmonium, should allow to fix the contribution of charmed-meson loops and allow an accurate determination of the light quark-mass ratio.
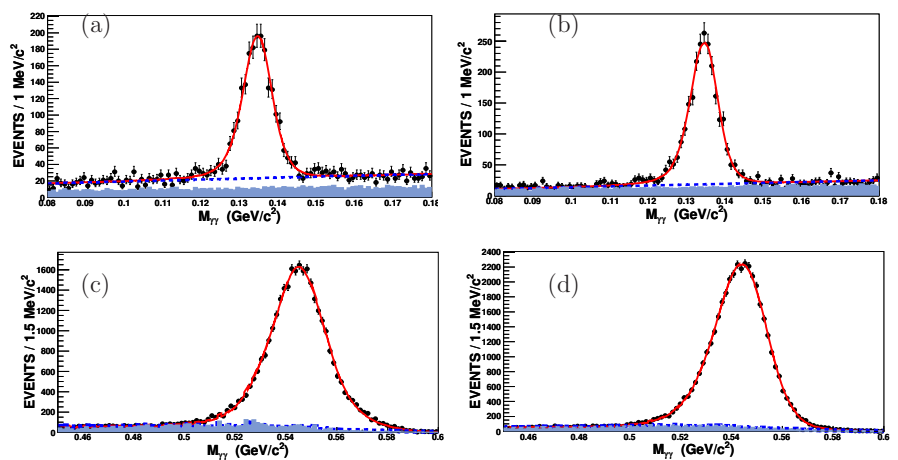

Figure 4: $M_{\gamma \gamma}$ distributions and fit results. (a) $\psi^{\prime} \rightarrow \pi^{\circ} J / \psi, J / \psi \rightarrow e^{+} e^{-}$, (b) $\psi^{\prime} \rightarrow \pi^{\circ} J / \psi, J / \psi \rightarrow \mu^{+} \mu^{-}$, (c) $\psi^{\prime} \rightarrow \eta J / \psi, J / \psi \rightarrow e^{+} e^{-}$, (d) $\psi^{\prime} \rightarrow \eta J / \psi, J / \psi \rightarrow \mu^{+} \mu^{-}$, where the points with error bars are data, and the red curves are the total fit results, and the dash curves are the fitted background shapes. The hatched histograms are the shapes of the sum of background events obtained from MC simulation and a side-band analysis.

\section{References}

[1] M. Ablikim et al. Nucl. Instrum. Meth. A 614, 345 (2010).

[2] M. Ablikim et al. (BESIII Collaboration) Phys. Rev. Lett. 104, 132002 (2010).

[3] M. Ablikim et al. (BESIII Collaboration), arXiv:1205.5103.

[4] K. Nakamura et al. (Particle Data Group), J. Phys. G 37, 075021 (2010).

[5] M. Ablikim et al. (BESIII Collaboration), Phys. Rev. Lett 108, 222002(2012).

[6] B.L. Ioffe, Yad. Fiz. 29, 1611(1979).

[7] J.F. Donoghue and S.F. Tuan, Phys. Lett. B 164, 401(1985). 
[8] B.L. Ioffe and M.A. Shifman, Phys. Lett. B 95, 99(1980).

[9] H. Mendez et al. (CLEO Collaboration), Phys. Rev. D 78, 011102 (2008).

[10] H. Leutwyler, Phys. Lett. B 374, 163(1996).

[11] F.K. Guo et al., Phys. Rev. Lett. 103, 082003(2009). 